\title{
Determination of the stretch tensor for structural transformations
}

\author{
Xian Chen ${ }^{\mathrm{a}, \mathrm{b}, \mathrm{c}}$, Yintao Song ${ }^{\mathrm{a}}$, Nobumichi Tamura ${ }^{\mathrm{b}}$, Richard D. James $^{\mathrm{a}}$ \\ ${ }^{a}$ Aerospace Engineering and Mechanics, University of Minnesota, Minneapolis, MN 55455 USA \\ ${ }^{b}$ Advanced Light Source, Lawrence Berkeley National Lab, CA 94702 USA \\ ${ }^{c}$ Mechanical and Aerospace Engineering, the Hong Kong University of Science and Technology, Clear Water \\ Bay, Hong Kong
}

\begin{abstract}
Structural transformations in crystalline solids are increasingly the basis of the functional behavior of materials. Recently, in diverse alloy systems, both low hysteresis and reversibility of phase transformations have been linked to the satisfaction of the nongeneric conditions of compatibility between phases. According to the Cauchy-Born rule, these conditions are expressed as properties of transformation stretch tensor. The transformation stretch tensor is difficult to measure directly due to the lack of knowledge about the exact transforming pathway during the structural change, and the complicating effects of microstructure. In this paper we give a rigorous algorithmic approach for determining the transformation stretch tensor from X-ray measurements of structure and lattice parameters. For some traditional and emerging phase transformations, the results given by the algorithm suggest unexpected transformation mechanisms.
\end{abstract}

Keywords: Phase Transformation, Geometrically Nonlinear Theory of Martensite, Crystallography, Lattice Correspondence

\section{Introduction}

Structural transformations increasingly underlie the unusual behavior of emerging functional materials designed for sensors/actuators [34, 26, 21], solid-state refrigerators [27, 13], batteries [20, 29], thermoelectrics [18] and direct energy conversion devices [39, 36]. These applications rely on the change of crystal structure. The resulting change of lattice parameters, together with sensitivity of magnetoelectric properties to lattice parameters, inspires an approach to the discovery of new functional phase-transforming materials [12, 43, 38, 37]. However, the formation of microstructure during phase transformation introduces elastic distortion at phase interfaces due to lattice misfit, which in turn leads to functional degradation. Recent studies of morphological interface compatibility for phase-transforming materials shed light on the effect of lowering and even eliminating this distortion by tuning composition so that the lattice parameters satisfy strong conditions of compatibility [8, 9]. These conditions are restrictions on the form of the transformation stretch tensor $[3,6]$ and the point groups of the two phases. The reversibility, thermal hysteresis, and resistance to cyclic degradation of functional materials have been dramatically linked to properties of the transformation stretch

Email address: james@umn.edu (Richard D. James) 
tensor and symmetries $[43,8,37,10,19]$. Here we propose an algorithmic approach for the determination of the transformation stretch tensor based on X-ray measurements, and we give a rigorous proof of its validity. The results below reveal some unexpected transformation mechanisms in traditional and emerging phase-transforming materials.

In principle, the determination of the transformation stretch tensor $\mathbf{U}$ is straightforward: simply observe where each atom goes during transformation and deduce the macroscale deformation gradient $\mathbf{F}$ associated to this motion. The stretch tensor associated to this macroscale deformation gradient (via the polar decomposition $\mathbf{F}=\mathbf{Q} \mathbf{U}, \mathbf{Q} \in \mathrm{SO}(3)$, $\mathbf{U}=\mathbf{U}^{T}$ positivedefinite), should the main quantity that is relevant to the stressed transition layers between phases that underlies improvements of reversibility discussed above.

In practice, however, determination of this macroscale deformation gradient is surprisingly subtle. First, not every atom is convected with this macroscopic deformation gradient. Rather, some sublattice of the parent (austenite) phase is transformed into some sublattice of the transformed (martensite) phase. The atoms within the unit cell of the chosen austenite sublattice are not generally deformed by this macroscale deformation gradient, but rather undergo independent movements called shuffing. Fix the lattices of austenite and martensite. It is easily seen that, by taking larger and larger sublattices of austenite and suitable choices of corresponding sublattices of martensite, the associated deformation gradient can be made arbitrarily close to the identity. Thus, the choices of sublattice are important.

Fortunately, there are guidelines for choosing the sublattice. The deformation gradient has been measured directly by macroscopic methods in a few cases by the so-called "two-surface analysis" $[25,15,33]$. This consists of scratching an austenite single crystal on two nonparallel surfaces, transforming the crystal to martensite by cooling, detwinning the crystal by stress to remove the inevitable microstructure that forms, carefully removing the stress, and measuring suitable lengths and angles associated to the scratches to get $\mathbf{F}$. In all of these cases of which we are aware it is found that relevant martensite sublattice is a primitive lattice of martensite ${ }^{1}$. The austenite sublattice is generally not primitive.

While the algorithm given below works in more general cases, it is here written to find the sublattice of austenite that is closest in a certain norm to the primitive lattice of martensite. The distance chosen is a measure of strain having certain algorithmic advantages. Besides being frame-indifferent, it also has certain advantages with regard to symmetry, i.e., lattices of different variants of the martensite phase have the same distance to the austenite sublattice. The idea of minimizing strain has a long history in martensite originating from the work of Bain [2].

A second consideration for the determination of $\mathbf{F}$ is well-known. This is the presence of lattice-invariant deformations. Referring to Fig. 1, suppose sublattice vectors of initial and final phases are, respectively, linearly independent vectors $\mathbf{a}_{i}$ and $\mathbf{b}_{i}$ for $i=1,2, \ldots, d$, where $d$ is the dimension of the lattice. A nonsingular linear transformation $\mathbf{F}: \mathbb{R}^{d} \rightarrow \mathbb{R}^{d}$ can be defined uniquely by

$$
\mathbf{F a}_{i}=\mathbf{b}_{i}, \quad i=1,2, \ldots, d
$$

\footnotetext{
${ }^{1} \mathrm{~A}$ primitive lattice of martensite is a sublattice of the martensite structure having a unit cell of smallest volume, i.e., it embodies the fundamental periodicity of the martensite lattice, accounting for the crystal structure and species.
} 

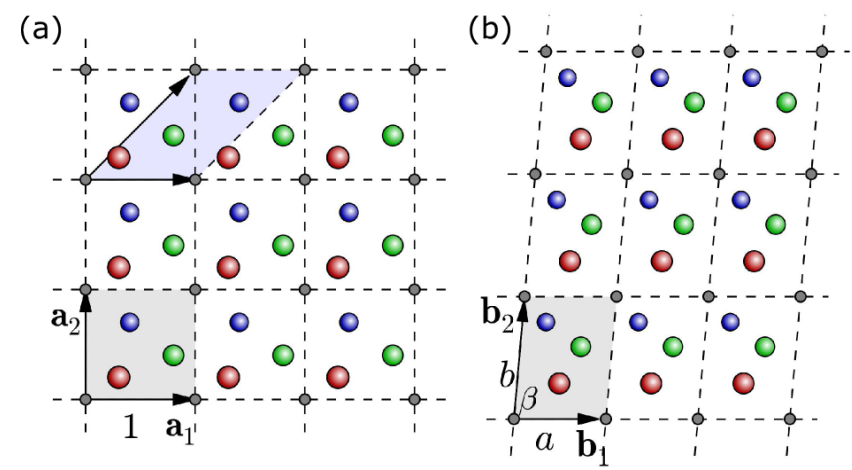

Figure 1: Non-uniqueness of Cauchy-Born deformation gradient from a (a) square lattice to (b) oblique lattice due to lattice invariant deformations. Red, blue and green balls represent different atomic species. Gray dots define the periodicity. In this example both lattices are chosen as primitive, for simplicity.

The notation $\mathbf{a}_{i} \rightarrow \mathbf{b}_{i}$ denotes a lattice correspondence. In the case of transformation in Fig. 1, one choice of the lattice correspondence can be $\mathbf{a}_{1} \rightarrow \mathbf{b}_{1}, \mathbf{a}_{2} \rightarrow \mathbf{b}_{2}$ where $\mathbf{a}_{1}=[1,0], \mathbf{a}_{2}=[0,1]$ and $\mathbf{b}_{1}=[a, 0]$ and $\mathbf{b}_{2}=[b \cos \beta, b \sin \beta]$ as illustrated in Fig. 1. The alternative set of vectors $\mathbf{a}_{1}$ and $\mathbf{a}_{1}+\mathbf{a}_{2}$ describes the same lattice (a), which results in a different correspondence from (a) to (b). This obviously changes the $\mathbf{F}$ and thus the transformation stretch tensor $\mathbf{U}$. More generally, any two sets of primitive lattice vectors for a given lattice (or, two sets of lattice vectors describing a sublattice) are related by a lattice invariant transformation (see, e.g., [35]) i.e., a unimodular matrix of integers. If we allow an invariant transformation for both initial and final phases, the ambiguity of $\mathbf{F}$ is $\mathbf{F} \rightarrow \Lambda_{(\mathrm{f})} \mathbf{F} \Lambda_{(\mathrm{i})}^{-1}$ where $\Lambda_{(\mathrm{i})}$ and $\Lambda_{(\mathrm{f})}$ denote the lattice invariant transformations for the initial and final lattices, respectively.

Experimental two-surface analysis is difficult for several reasons, beginning with the requirement of a large oriented single crystal. A more common experimental approach is first to infer the lattice correspondence, which effectively says which atoms go where (for a subset of atoms), and from this information and the precise crystallography, to deduce the transformation stretch tensor. This is done indirectly by X-ray diffraction methods. The most advanced procedure is to use synchrotron Laue microdiffraction techniques, so as to avoid the need to prepare a single crystal, together with a heating/cooling stage (Section 6). One sees that, during transformation, some spots associated to a zone-axis move just a small distance. It is then plausible to assume that the lattice planes associated to these pairs of spots are transformed one to another. If this information is known for a sufficient number of planes, a plausible lattice correspondence can be postulated, and the stretch tensor can be determined.

In Section 6 we review this procedure. From our study of examples in Section 5 we find that for the cubic-to-monoclinic transformation in $\mathrm{Zn}_{45} \mathrm{Au}_{30} \mathrm{Cu}_{25}$ the algorithm unexpectedly gives two transformation stretch tensors, associated to two different correspondences, that have comparable strain. (Note: the algorithm gives the smallest, next smallest, ..., etc.) One of them was not known previously, illustrating that the algorithm can be used for the discovery of plausible transformation mechanisms. With this information we then show explicitly the micro-Laue diffraction patterns for this alloy (Section 6). We show from these patterns that neither of the two mechanisms is favored by the typical experimental procedure described in the preceding paragraph. Of course, this could indicate that both mechanisms occur in the alloy, which would be especially interesting. This example and the theoretical example of 
Fig. 3 suggest that this algorithm can be useful to reveal unexpected but highly plausible new mechanisms. We also note that algorithm can be used effectively in conjunction with these micro-Laue diffraction methods.

\section{Lattice correspondence for structural transformation}

Based on a natural intuition that "a mode of atomic shift requires minimum motion", Bain [2] proposed the now famous lattice correspondence for the formation of bcc $\alpha$-Fe from fcc $\gamma-\mathrm{Fe}$,

$$
\begin{aligned}
& {[100]_{\mathrm{fcc}} \rightarrow[110]_{\mathrm{bcc}}} \\
& {[010]_{\mathrm{fcc}} \rightarrow[\overline{1} 10]_{\mathrm{bcc}}} \\
& {[001]_{\mathrm{fcc}} \rightarrow[001]_{\mathrm{bcc}} .}
\end{aligned}
$$

In this original article there is little justification beyond the statements, "If one regards the centers of faces as the corners of a new unit, a body-centered structure is already at hand" and the assertion that the proposed mechanism is "the only easy method of constructing a body-centered atomic structure from the fact-centered cubic crystal" [2]. Bain did not give an explicit expression what he meant by minimum motion, and so could not give a proof that the Bain correspondence given by (2) gives the least transformation strain for the fccbcc transformation. The applicability of the Bain or near-Bain strain (or its inverse) and associated correspondence to many transformations was suggested by Bowles and Wayman [7]. Following these authors, we continue to use the terminology "Bain correspondence" for this correspondence, allowing for small perturbations of the bec or fcc .

The Bain correspondence has been applied to study numerous phase transformations such as Fe-Pt alloy [40], equal-atomic NiTi [32, 22] and $\mathrm{CuAlNi}$ [11]. It is also widely used for modeling transformation pathways and formation of microstructure in reversible phase transformations $[4,6,17]$. Indeed, most of these studied transformations are not exactly the same as $\gamma$-Fe to $\alpha$-Fe, but they all follow a transformation analogy that a cubic-type structure (i.e., simple cubic, fcc or bcc type) deforms to a structure with equal or lower symmetry.

The martensitic phases of these near-Bain transformations are usually perturbed body or face-centered sublattices of the austenite phase, schematically shown as Fig. 2. To illustrate the effect of this perturbation quantitatively, we construct a structural transformation from a bcc lattice with $a_{0}=1$ to a monoclinic lattice with $a=0.961, b=1.363, c=1.541$, and $\beta=97.78^{\circ}$. Fig. 3(a) shows the bcc lattice with two sublattice unit cells (red and blue). Conventional wisdom would say that the Bain correspondence (red $\rightarrow$ black in Fig. 3(c)) is appropriate for this transformation. However, our algorithm proposed in this paper reveals an unexpected alternative correspondence (blue $\rightarrow$ black, Fig. 3(b)). Both red and blue unit cells contain 4 lattice points $(n=4)$, and are similar to the monoclinic primitive cell in size and shape. Fig. 3(b) and (c) shows the comparison of lattice distortions. Both mechanisms give exactly the same final monoclinic sublattice (in black). However, by quantitative calculation, the principal stretches for the new correspondence are all closer to 1 than for the Bain correspondence. More precisely, both correspondences have the same middle principal stretch, and the other principal stretches are $\lambda_{1}=0.9291, \lambda_{3}=1.1167$ for the new correspondence and $\lambda_{1}=0.9283, \lambda_{3}=1.1176$ for the Bain correspondence.

The significance of finding the correct lattice correspondence for a structural transformation is emphasized in the literature [42, 7]. The problem was well-appreciated by Lomer [28] 


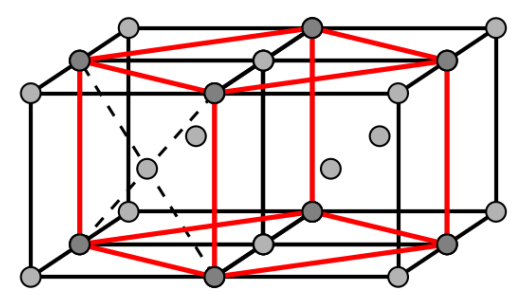

(a)

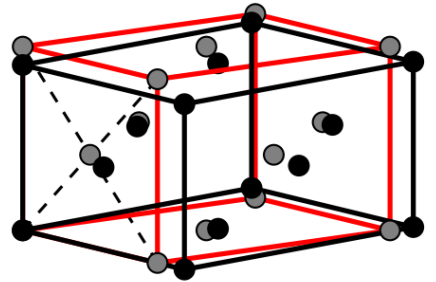

(b)

Figure 2: A Bain correspondence from bcc to monoclinic: (a) an orthogonal sublattice of bcc (red); (b) the deformed monoclinic lattice (black) from the orthogonal face-centered sublattice.

(a)

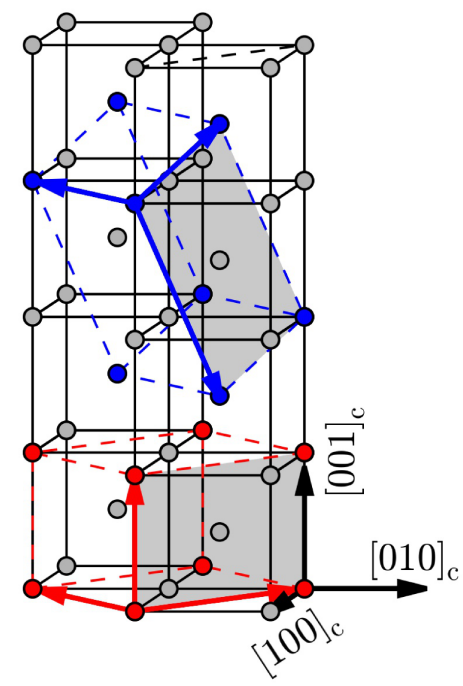

(b)

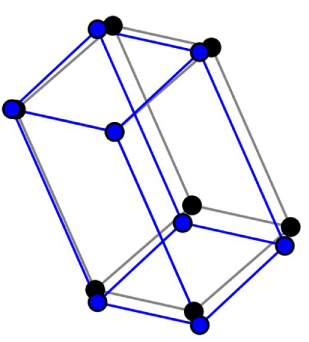

(c)

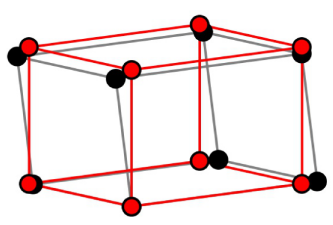

Figure 3: A comparison of the Bain correspondence and a new correspondence found using the algorithm presented in this paper. (a) The bcc lattice and two of its sublattices (red and blue) of size 4. Lattice distortions between these sublattices and the deformed lattices (b) and (c) (black; for clarity atoms within the unit cell are not shown). See the text for further information. 
as early as the mid-1950s. In his study of the mechanism of the $\beta \rightarrow \alpha$ phase transformation of $\mathrm{U}_{98.6} \mathrm{Cr}_{1.4}$, he examined theoretically (by hand) 1,600 possible transformation mechanisms, and reduced this to three correspondences having the smallest principal strains, which he considered the likely candidates.

Direct experimental measurement of the macroscopic finite strain of transformation together with accurate structural characterization by X-ray diffraction provides a possible way to determine the lattice correspondence and thus the transformation stretch tensor. But, as indicated above, this is technically difficult due to (i) the need for an oriented single crystal, (ii) the need to remove the inevitable fine microstructures that form during transformation due to constraints of compatibility, and (iii) the need for an accurate measure of full finite strain tensor. Even using a state-of-art TEM on a pre-oriented single crystal sample, one can not definitively remove the ambiguities among many lattice correspondences due to some inevitable obstacles: tracking the evolution of diffraction spots in a fast structural transformation process, simultaneously indexing both phases, and most significantly, finding a special zone that can unambiguously reveal the differences among various lattice correspondences. This difficulty is discussed below for a particular example in Section 6 .

Consider a Bravais lattice $\mathcal{L}=\left\{\sum n^{i} \mathbf{e}_{i}: n^{1}, \ldots n^{d} \in \mathbb{Z}^{d}\right\}$ determined by linearly independent lattice vectors $\mathbf{e}_{1}, \ldots, \mathbf{e}_{d} \in \mathbb{R}^{d}, i=1, \ldots, d$, and assemble the lattice vectors as the columns of a $d \times d$ matrix $\mathbf{E}=\left(\mathbf{e}_{1}, \ldots, \mathbf{e}_{d}\right) . \mathcal{L}$ can equivalently be denoted

$$
\mathcal{L}=\mathcal{L}(\mathbf{E})=\left\{\mathbf{r} \in \mathbb{R}^{d}: \mathbf{r}=\mathbf{E} \boldsymbol{\xi}, \boldsymbol{\xi} \in \mathbb{Z}^{d}\right\} .
$$

This determinant is the ( $d$-dimensional) volume of a unit cell of $\mathcal{L}(\mathbf{E})$.

Given two lattices $\mathcal{L}(\mathbf{E})$ and $\mathcal{L}\left(\mathbf{E}^{\prime}\right)$, the $d \times d$ nonsingular matrix $\mathbf{L}$ satisfying $\mathbf{E}^{\prime}=\mathbf{E L}$ is called a correspondence matrix from $\mathcal{L}(\mathbf{E})$ to $\mathcal{L}\left(\mathbf{E}^{\prime}\right)$. As noted above, the two lattices $\mathcal{L}(\mathbf{E})$ and $\mathcal{L}\left(\mathbf{E}^{\prime}\right)$ are the same if and only if a correspondence matrix $\mathbf{L}$ is a unimodular matrix of integers, or, briefly, $\mathbf{L} \in G L(d, \mathbb{Z})$. If a correspondence matrix $\mathbf{L}$ is a matrix of integers with $|\operatorname{det} \mathbf{L}|>1$, then $\mathcal{L}\left(\mathbf{E}^{\prime}\right)$ is a sublattice of $\mathcal{L}(\mathbf{E})$. The quantity $|\operatorname{det} \mathbf{L}|$ is the volume ratio of the unit cell of $\mathcal{L}\left(\mathbf{E}^{\prime}\right)$ to that of $\mathcal{L}(\mathbf{E})$.

Correspondence matrices are often reported for conventional rather than primitive descriptions of a given Bravais lattice, particularly for 7 of the 14 types of Bravais lattices in 3D. For example, with $d=3$ the conventional description for an fcc lattice with lattice parameter $a_{0}$ is an orthogonal basis, so $\mathbf{E}_{\mathrm{conv}}=a_{0} \mathbf{I}=\mathbf{E} \boldsymbol{\chi}$, where, for example,

$$
\mathbf{E}=\frac{a_{0}}{2}\left[\begin{array}{lll}
1 & 0 & 1 \\
1 & 1 & 0 \\
0 & 1 & 1
\end{array}\right], \quad \chi=\left[\begin{array}{ccc}
1 & 1 & -1 \\
-1 & 1 & 1 \\
1 & -1 & 1
\end{array}\right]
$$

Here, $\operatorname{det} \boldsymbol{\chi}=4$ so the volume of the conventional unit cell is 4 times that of the primitive cell. From now on, the symbol $\chi$ is reserved for a correspondence matrix from the primitive to conventional unit cell of a Bravais lattice: $\mathbf{E}_{\mathrm{conv}}=\mathbf{E} \boldsymbol{\chi}$. The change from the primitive to the conventional correspondence is merely a notational change, but it is important when comparing with correspondences in the literature. The use of the conventional description is only done at the last stage, after implementation of the algorithm.

Let $\mathbf{E}_{A}=\left(\mathbf{a}_{1}, \ldots, \mathbf{a}_{d}\right)$ and $\mathbf{E}_{B}=\left(\mathbf{b}_{1}, \ldots, \mathbf{b}_{d}\right)$. We seek a sublattice of the austenite primitive lattice $\mathcal{L}\left(\mathbf{E}_{A}\right)$ that is mapped to the martensite primitive lattice of $\mathcal{L}\left(\mathbf{E}_{B}\right)$. Note that we fix 
the primitive lattice of martensite in applications of the algorithm, since this gives interesting answers. The algorithm is however not restricted to this hypothesis. For example, it can also handle cases in which a sublattice of the martensite lattice is chosen, or all sublattices of bounded volume with a given bound. Let $\ell \in \mathbb{Z}^{d \times d}$ be the correspondence matrix giving the sublattice $\mathcal{L}\left(\mathbf{E}_{A} \boldsymbol{\ell}\right)$ that is mapped to the final lattice $\mathcal{L}\left(\mathbf{E}_{B}\right)$ during the transformation. The basic equation (1) in this case becomes $\mathbf{F} \mathbf{E}_{A} \boldsymbol{\ell}=\mathbf{E}_{B}$, and the transformation stretch tensor $\mathbf{U}$ is the unique positive-definite square root of $\mathbf{F}^{T} \mathbf{F}$.

\section{Distance Function}

We introduce the following function as a measure of the distance from $\mathbf{U}$ to $\mathbf{I}$ :

$$
\begin{aligned}
\operatorname{dist}\left(\boldsymbol{\ell}, \mathbf{E}_{A}, \mathbf{E}_{B}\right) & =\left\|\left(\mathbf{F}^{T} \mathbf{F}\right)^{-1}-\mathbf{I}\right\|^{2} \\
& =\left\|\mathbf{E}_{A} \boldsymbol{\ell} \mathbf{E}_{B}^{-1} \mathbf{E}_{B}^{-T} \boldsymbol{\ell}^{T} \mathbf{E}_{A}^{T}-\mathbf{I}\right\|^{2} .
\end{aligned}
$$

$\|\cdot\|$ denotes the Frobenius norm, $\|\mathbf{A}\|=\sqrt{\operatorname{tr} \mathbf{A}^{T} \mathbf{A}}$.

The distance (3) is independent of rigid rotations of both lattices, and is particularly attractive from the point of view of symmetry. Physically, it represents the Lagrangian strain of the structural transformation. The use of inverse of $\mathbf{F}^{T} \mathbf{F}$ avoids possible noninvertibility of $\boldsymbol{\ell}$ that may arise during the minimization process. In addition, this norm is exactly preserved by point group transformations of both Bravais lattices. That is, if orthogonal tensors $\mathbf{R}_{A}$ and $\mathbf{R}_{B}$ are, respectively, in the point groups of $\mathcal{L}\left(\mathbf{E}_{A}\right)$ and $\mathcal{L}\left(\mathbf{E}_{B}\right)$, i.e., $\mathcal{L}\left(\mathbf{E}_{A}\right)=\mathcal{L}\left(\mathbf{R}_{A} \mathbf{E}_{A}\right)$ and $\mathcal{L}\left(\mathbf{E}_{B}\right)=\mathcal{L}\left(\mathbf{R}_{B} \mathbf{E}_{B}\right)$, there exist associated matrices $\boldsymbol{\mu}_{A}$ and $\boldsymbol{\mu}_{B}$ such that $\mathbf{R}_{A} \mathbf{E}_{A}=\mathbf{E}_{A} \boldsymbol{\mu}_{A}$ and $\mathbf{R}_{B} \mathbf{E}_{B}=\mathbf{E}_{B} \boldsymbol{\mu}_{B}$ then the distance transforms as

$$
\operatorname{dist}\left(\boldsymbol{\mu}_{A} \boldsymbol{\ell} \boldsymbol{\mu}_{B}, \mathbf{E}_{A}, \mathbf{E}_{B}\right)=\operatorname{dist}\left(\boldsymbol{\ell}, \mathbf{E}_{A}, \mathbf{E}_{B}\right) .
$$

Note that $\boldsymbol{\mu}_{A, B}$ are integral matrices of determinant \pm 1 , so $\operatorname{det} \boldsymbol{\ell}=\operatorname{det} \boldsymbol{\mu}_{A} \boldsymbol{\ell} \boldsymbol{\mu}_{B}$. Thus, immediately one minimizer of the distance with assigned determinant gives the expected symmetryrelated minimizers. Alternative distances are analyzed by Koumatos and Mühlemann [30].

As noted above it is typical to report the correspondence matrix in terms of the conventional basis instead of the primitive one. If $\boldsymbol{\ell}^{*}$ is a minimizer of $\operatorname{dist}\left(\boldsymbol{\ell}, \mathbf{E}_{A}, \mathbf{E}_{B}\right)$ the conversion is done by $\mathbf{L}^{*}=\chi_{A}^{-1} \ell^{*} \chi_{B}$. Note that $\mathbf{L}^{*}$ is not necessarily a matrix of integers.

A significant property of the distance function (3) will be used to justify our algorithm below. Fixing $\mathbf{E}_{A}$ and $\mathbf{E}_{B}$, the distance function can be trivially extended to a function over real matrices, $f(\mathbf{L})=\operatorname{dist}\left(\mathbf{L}, \mathbf{E}_{A}, \mathbf{E}_{B}\right)$. Denoting $\mathbf{X}_{L}=\mathbf{E}_{A} \mathbf{L} \mathbf{E}_{B}^{-1} \mathbf{E}_{B}^{-T} \mathbf{L}^{T} \mathbf{E}_{A}^{T}$ and using $\mathbf{X}_{L} \cdot \mathbf{I} \leq\left\|\mathbf{X}_{L}\right\|\|\mathbf{I}\|=\sqrt{3}\left\|\mathbf{X}_{L}\right\|$, we have

$$
\begin{aligned}
f(\mathbf{L}) & =\left\|\mathbf{X}_{L}\right\|^{2}-2 \mathbf{X}_{L} \cdot \mathbf{I}+3 \\
& \geqslant\left\|\mathbf{X}_{L}\right\|^{2}-2 \sqrt{3}\left\|\mathbf{X}_{L}\right\|+3=\left(\left\|\mathbf{X}_{L}\right\|-\sqrt{3}\right)^{2},
\end{aligned}
$$

Choose any integral matrix $\boldsymbol{\ell}_{1}$ and define $C_{1}=f\left(\boldsymbol{\ell}_{1}\right)$. By (5) the minimizer(s) of $f(\mathbf{L})$ necessarily lie in the bounded set $\left\|\mathbf{X}_{L}\right\| \leq \sqrt{3}+\sqrt{C_{1}}$, that is, $\left\|\mathbf{X}_{L}\right\|^{2} \leq 3+C_{1}+2 \sqrt{3 C_{1}}$. Let $\alpha$ be the minimum of $\left\|\mathbf{X}_{L}\right\|^{2}$ under the constraint $\|\mathbf{L}\|=1$, then we have

$$
\alpha\|\mathbf{L}\|^{4} \leqslant\left\|\mathbf{X}_{L}\right\|^{2}<3+C_{1}+2 \sqrt{3 C_{1}} .
$$

That is, all the $\mathbf{L}$ 's such that $f(\mathbf{L})<C_{1}$ live in the sphere with the radius of $\left(\left(3+C_{1}+\right.\right.$ $\left.\left.2 \sqrt{3 C_{1}}\right) / \alpha\right)^{1 / 4}$ in $\mathbb{R}^{9}$. 

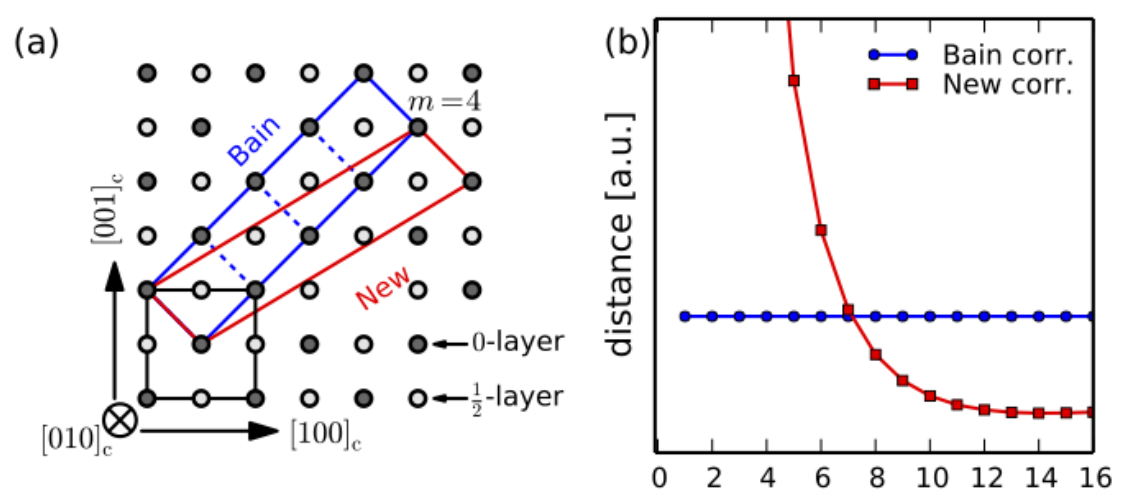

Figure 4: A change of correspondence detected by the algorithm as the size of the martensite sublattice increases. Lattice parameters are given in the text. (a) (010) projection of the fcc lattice with two unit cells (red and blue) of a sublattice corresponding to $m=4$. The graph (b) summarizes the result of running the algorithm with the choices of $m=1, \ldots, 16$ and, for each $m$, fixing the sublattice of size $m$ to be the martensite sublattice. In (b) the resulting values of the distance function are plotted, and the overall minimizing value of distance is the minimum of the two graphs at each $m$. For clarity the deformed configurations are not shown.

\section{Algorithm for structural phase transformation}

The algorithm for the determination of the $N$ best lattice correspondences and their associated transformation stretch tensors is given below. As explained above, $\mathbf{E}_{B}$ is given.

1. Calculate the primitive bases and the conversion matrices for the conventional cells from the input lattice parameters: $\mathbf{E}_{\mathrm{A}, \mathrm{B}}$ and $\boldsymbol{\chi}_{\mathrm{A} \text {, B }}$. Calculate $\alpha$ by minimizing the term $\mathbf{X}_{L}$ with respect to $\mathbf{L}$ for all $\|\mathbf{L}\|=1$.

2. Choose $N$ integral matrices $\boldsymbol{\ell}_{i}, i=1, \ldots, N$ as the initial guess of the solution list such that $\operatorname{det} \boldsymbol{\ell}_{i}$ is close to $\operatorname{det} \mathbf{E}_{B} / \operatorname{det} \mathbf{E}_{A}$ and $\operatorname{dist}\left(\boldsymbol{\ell}_{i}, \mathbf{E}_{A}, \mathbf{E}_{B}\right)$ is small.

3. Let $C_{1}$ be the maximum $f\left(\boldsymbol{\ell}_{i}\right)$ for $\boldsymbol{\ell}_{i}$ 's in the solution list and calculate the radius $\rho=\left(\left(3+C_{1}+2 \sqrt{3 C_{1}}\right) / \alpha\right)^{1 / 4}$.

4. Calculate iteratively the distance of integral matrices in the sphere of radius $\rho$. If a new matrix (not in the current solution list) with smaller distance is detected, update the solution list, recalculate the radius, and repeat this step.

5. For each solution $\boldsymbol{\ell}_{i}$, calculate the Cauchy-Born deformation gradient $\mathbf{F}_{i}=\mathbf{E}_{B}\left(\mathbf{E}_{A} \boldsymbol{\ell}_{i}\right)^{-1}$ and the transformation stretch tensor $\mathbf{U}_{i}=\left(\mathbf{F}_{i}^{T} \mathbf{F}_{i}\right)^{1 / 2}$. Finally, rewrite all the solutions in the conventional bases: $\mathbf{L}_{i}^{*}=\chi_{\mathrm{A}}^{-1} \boldsymbol{\ell}_{i} \boldsymbol{\chi}_{\mathrm{B}}$.

The algorithm converges in a finite number of steps and gets all matrices with the $N$ lowest distances (up to the degeneracy (4)) because it searches through all matrices of integers satisfying the rigorous bounds (6). The power $1 / 4$ in the definition of $\rho$ is particularly advantageous for numerical reasons.

In Fig. 4 we give an example computed by the algorithm that reveals a switch from Bain correspondence to a new correspondence with increasing lattice complexity measured by the modulation $m$ [31]. Consider a transformation from an fcc lattice with lattice parameter $a_{0}=2$ to a monoclinic lattice with realistic lattice parameters $a=1.41, b=1.99, c=$ $1.42 m, \beta=86^{\circ}$, where the integer $m>0$ denotes the modulation along monoclinic $c$-axis. 
Fig. 4(a) shows the undeformed fcc lattice projected onto the (010) plane. Fig. 4(b) shows the change in distance function for the two correspondences with $m$ varying from 1 to 16 . Initially, the Bain correspondence is much smaller than the new one, however it loses its privilege after the 7th modulation. The results suggest that both kinds of lattice correspondence are feasible in a structural transformation for some special lattice parameters, and in this case $m=7$ is the critical case. These long stacking period structures are common especially for the adaptive martensite structures [21].

\section{Examples}

As a first example we remark briefly about the classical Bain mechanism for bcc $\rightarrow$ fcc. Our algorithm proves that with the choice of distance we have made, the Bain correspondence gives the smallest distance $(d=0.277$ for $\alpha$-Fe to $\gamma$-Fe) between these primitive lattices. The next smallest is the rather large number $d=2.74$ suggesting that the Bain correspondence is strongly preferred in this case. By way of comparison, the case presented in Fig. 3 has $d=$ 0.0702 for the smallest solution and $d=0.0713$ for the next smallest solution. A fascinating consequence of our algorithm is that if the fcc in the example bcc $\rightarrow$ fcc is perturbed slightly to monoclinic, and a modest size sublattice of this monoclinic lattice is given (instead of the primitive lattice), then the situation changes drastically, i.e., there can be modest size sublattices and non-Bain mechanisms that give a smaller strain than that given by the Bain correspondence (cf., Fig. 3)

Table 1: Transformation principal stretches, the associated lattice correspondences and derived orientation relationships for various phase-transforming materials

\begin{tabular}{|c|c|c|c|}
\hline materials & principal stretches & lattice correspondence & predicted orientation relationship \\
\hline \multirow{2}{*}{$\begin{array}{l}\mathrm{Zn}_{45} \mathrm{Au}_{30} \mathrm{Cu}_{25}[37] \\
\quad \mathrm{L} 2_{1} \rightarrow \mathrm{M} 18 \mathrm{R}\end{array}$} & $\begin{array}{l}0.9363 \\
1.0017 \\
1.0589\end{array}$ & 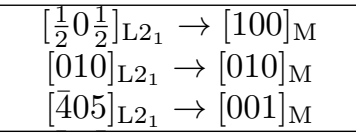 & 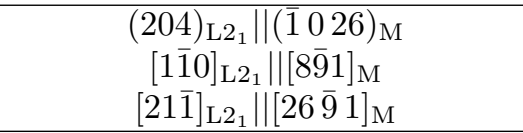 \\
\hline & $\begin{array}{l}0.9363 \\
1.0006 \\
1.0600\end{array}$ & $\begin{aligned} {\left[\frac{1}{2} 0 \frac{1}{2}\right]_{\mathrm{L} 2_{1}} } & \rightarrow[100]_{\mathrm{M}} \\
{[010]_{\mathrm{L} 2_{1}} } & \rightarrow[010]_{\mathrm{M}} \\
{\left[\frac{9}{2} 0 \frac{9}{2}\right]_{\mathrm{L} 2_{1}} } & \rightarrow[001]_{\mathrm{M}}\end{aligned}$ & $\begin{array}{c}(204)_{\mathrm{L} 2_{1}} \|(\overline{1} 027)_{\mathrm{M}} \\
{[1 \overline{1} 0]_{\mathrm{L} 2_{1}} \|[9 \overline{9} 1]_{\mathrm{M}}} \\
{[21 \overline{1}]_{\mathrm{L} 2_{1}} \|[27 \overline{9} 1]_{\mathrm{M}}}\end{array}$ \\
\hline $\begin{array}{l}\mathrm{CuAl}_{30} \mathrm{Ni}_{4}[33] \\
\quad \beta_{1} \rightarrow \gamma^{\prime}\end{array}$ & $\begin{array}{l}0.9178 \\
1.0231 \\
1.0619\end{array}$ & $\begin{aligned} {\left[\frac{1}{2} 0 \frac{1}{2}\right]_{\beta_{1}} } & \rightarrow[100]_{\gamma^{\prime}} \\
{[010]_{\beta_{1}} } & \rightarrow[010]_{\gamma^{\prime}} \\
{\left[\frac{1}{2} 0 \frac{1}{2}\right]_{\beta_{1}} } & \rightarrow[001]_{\gamma^{\prime}}\end{aligned}$ & $\begin{array}{l}(110)_{\beta_{1}} \|(121)_{\gamma^{\prime}} \\
{[1 \overline{1} \overline{1}]_{\beta_{1}} \|[2 \overline{1} 0]_{\gamma^{\prime}}}\end{array}$ \\
\hline $\begin{array}{c}\mathrm{Ti}_{95} \mathrm{Mn}_{5}[23] \\
\text { bcc } \rightarrow \text { hexagonal }\end{array}$ & $\begin{array}{l}0.9052 \\
1.0164 \\
1.1086\end{array}$ & $\begin{aligned} {[010]_{\mathbf{c}} } & \rightarrow[100]_{\mathbf{h}} \\
{\left[\frac{1}{2} \frac{1}{2} \frac{1}{2}\right]_{\mathbf{c}} } & \rightarrow[010]_{\mathbf{h}} \\
{[101]_{\mathbf{c}} } & \rightarrow[001]_{\mathbf{h}}\end{aligned}$ & $\begin{array}{l}(1 \overline{1} \overline{1})_{\mathbf{c}} \|(214)_{\mathbf{h}} \\
{[\overline{1} \overline{2} 1]_{\mathbf{c}} \|[20 \overline{1}]_{\mathbf{h}}}\end{array}$ \\
\hline $\begin{array}{l}\mathrm{Ru}_{50} \mathrm{Nb}_{50}[16] \\
\quad \beta^{\prime} \rightarrow \beta^{\prime}\end{array}$ & $\begin{array}{l}0.9791 \\
1.0024 \\
1.0169\end{array}$ & $\begin{aligned} {[11 \overline{2}]_{\beta^{\prime}} } & \rightarrow[100]_{\beta^{\prime \prime}} \\
{[1 \overline{1} 0]_{\beta^{\prime}} } & \rightarrow[010]_{\beta^{\prime \prime}} \\
{[11 \overline{1}]_{\beta^{\prime}} } & \rightarrow[001]_{\beta^{\prime \prime}}\end{aligned}$ & $\begin{array}{l}(100)_{\beta^{\prime}} \|(111)_{\beta^{\prime \prime}} \\
{[011]_{\beta^{\prime}} \|[1 \overline{1} 0]_{\beta^{\prime \prime}}}\end{array}$ \\
\hline $\begin{array}{l}\mathrm{Sb}_{2} \mathrm{Te}_{3} / \mathrm{PbTe}[8] \\
\mathrm{fcc} \rightarrow \text { hexagonal }\end{array}$ & $\begin{array}{l}0.9384 \\
0.9384 \\
1.0779\end{array}$ & $\begin{array}{l}{\left[\frac{1}{2} \frac{1}{2} 0\right]_{\mathbf{c}} \rightarrow[100]_{\mathbf{h}}} \\
{\left[0 \frac{1}{2} \frac{1}{2}\right]_{\mathbf{c}} \rightarrow[010]_{\mathbf{h}}} \\
{[2222]_{\mathbf{c}} \rightarrow[001]_{\mathbf{h}}}\end{array}$ & $\begin{array}{l}(\overline{1} 10)_{\mathbf{c}} \|(010)_{\mathbf{h}} \\
{[001]_{\mathbf{c}} \|[\overline{4} 8 \overline{1}]_{\mathbf{h}}}\end{array}$ \\
\hline $\begin{array}{l}\text { Terephthalic acid }[1] \\
\text { triclinic I } \rightarrow \text { triclinic II }\end{array}$ & $\begin{array}{l}0.8244 \\
0.9373 \\
1.3424\end{array}$ & $\begin{array}{l}{[012]_{\mathbf{I}} \rightarrow[100]_{\mathbf{I I}}} \\
{[110]_{\mathbf{I}} \rightarrow[010]_{\mathbf{I I}}} \\
{[001]_{\mathbf{I}} \rightarrow[001]_{\mathbf{I I}}}\end{array}$ & $\begin{array}{l}{[100]_{\mathbf{I}}||[112]_{\mathbf{I I}}} \\
{[010]_{\mathbf{I}}||[102]_{\mathbf{I I}}} \\
{[110]_{\mathbf{I}}||[010]_{\mathbf{I I}}}\end{array}$ \\
\hline
\end{tabular}

Table 1 shows the results calculated by the algorithm for six materials. The types of 
transformation are chosen to be diverse. In all cases except $\mathrm{Zn}_{45} \mathrm{Au}_{30} \mathrm{Cu}_{25}$ (see below) the algorithm gives the accepted orientation relationship.

Among these examples, we list two solutions for $\mathrm{Zn}_{45} \mathrm{Au}_{30} \mathrm{Cu}_{25}$. The material has been recently found to satisfy the cofactor conditions [9], which have been shown [37] to promote unusually low thermal hysteresis $\left(\approx 2^{\circ} \mathrm{C}\right)$ and enhanced reversibility, owing to a fluid-like flexible martensite microstructure. It was believed [37] to transform by the second solution, Table 1. However, the table shows that the first solution is the one having the smallest distance and the smallest transformation strain by various measures. Coincidentally, the new transformation stretch tensor also satisfies closely the cofactor conditions. To investigate this further, the same sample of $\mathrm{Zn}_{45} \mathrm{Au}_{30} \mathrm{Cu}_{25}$ used in [37] was characterized by synchrotron X-ray Laue microdiffraction. The results are discussed in the next section.

Note that the algorithm is applicable to a wide range of phase transformations even if the initial and final crystal structures do not have a group/sub-group relation. Examples are $\mathrm{Ti}_{95} \mathrm{Mn}_{5}$ and $\mathrm{Sb}_{2} \mathrm{Te}_{3} / \mathrm{PbTe}$ (Table 1), which are not reversible. The principal stretches of $\mathrm{Sb}_{2} \mathrm{Te}_{3}$ as well as the lattice correspondence reported here are consistent with the experimental results in reference [8]. The algorithm can be also applied to organic materials when the molecular chains have sufficient periodicity. One extreme example is the polymorphic transformation between two triclinic lattices in terephthalic acid (see Table 1). In this case the calculated principal stretches agree with the macroscopic deformation [5] of the polymorphic transformation of this material as shown in Fig. 5 [1, 14].

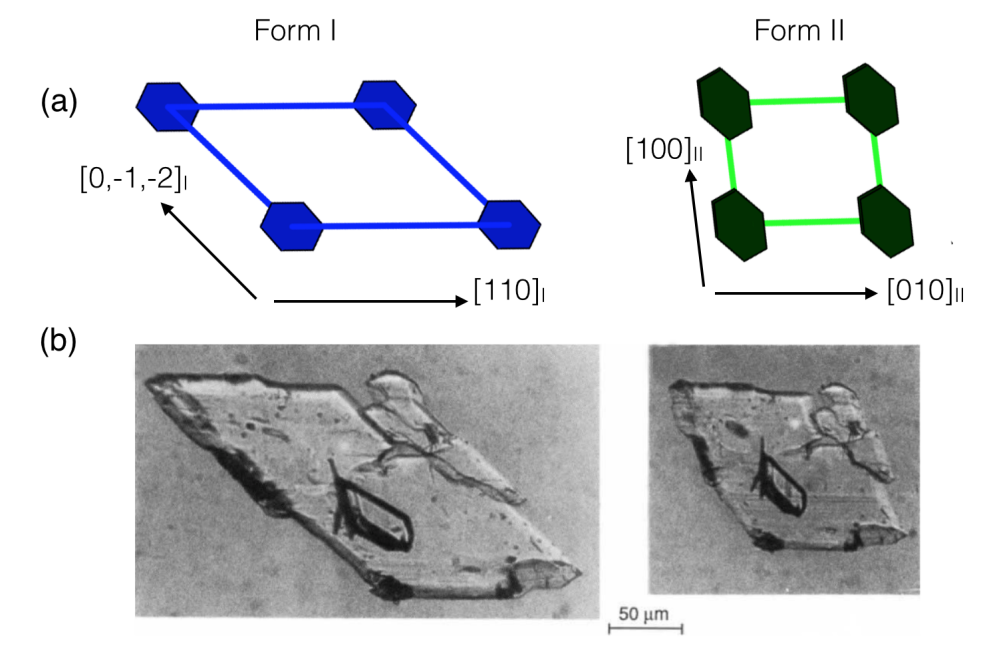

Figure 5: The macroscopic deformation in terephthalic acid during polymorphic transformation predicted by our algorithm: (a) deformation associated to the predicted transformation stretch tensor; (b) the observed morphologic change [14] between two polymorphs of the material shown viewed (approximately) along the same axis as in (a) (The terminology for forms I and II is that of M. Bailey and C. Brown [1]; note that the opposite terminology is used by R. J. Davey et al. [14]). 


\section{X-ray microdiffraction experiment on $\mathrm{Zn}_{45} \mathrm{Au}_{30} \mathrm{Cu}_{25}$}

\subsection{Physical properties and crystal structures}

The transformation temperature for $\mathrm{Zn}_{45} \mathrm{Au}_{30} \mathrm{Cu}_{25}$ is about $-40^{\circ} \mathrm{C}$ measured by Differential Scanning Calorimetry [37]. The austenite phase is face-centered cubic (L2 1 ) with lattice parameter $a_{0}=6.1606 \AA$, whereas the martensite phase is monoclinic with 18 atomic layer modulation along the crystallographic c-axis. The lattice parameters of martensite are $a=4.4580 \AA$, $b=5.7684 \AA$ A,$c=40.6980 \AA$ A $\beta=86.80^{\circ}[37]$.

\subsection{Experiment and results}

The experiment was conducted at the Advanced Light Source BL 12.3.2 X-ray Laue Microdiffraction, Lawrence Berkeley National Laboratory. For accurate determination of the structural parameters and the investigation of transformation pathways, we used a Domed Hot Stage (DHS 900) for in-situ measurement of the structural change by two diffraction methods (polychromatic and monochromatic) while slowly varying the temperature. The Laue patterns (by polychromatic diffraction) were continuously collected per $1^{\circ} \mathrm{C}$ for a complete transformation loop [24]. This temporal-scan gives the orientational dynamics of the phase transformation. Fig. 6 shows the Laue patterns corresponding to pure austenite, mixed phases and pure martensite, respectively. The spots in Laue pattern represent the diffracted crystallographic planes. We used the XMAS (X-ray Microdiffraction Analysis Software [41]) to obtain the orientation matrix by indexing the Laue pattern based on the lattice parameters and periodicities given in section 6.1. The austenite orientation matrix $\mathbf{O}_{a}$ for Fig. 6(a) and martensite orientation matrix $\mathbf{O}_{m}$ for Fig. 6(c) are

$$
\mathbf{O}_{a}=\left[\begin{array}{ccc}
0.6103 & -0.0689 & 0.0477 \\
0.0630 & 0.6085 & 0.0728 \\
-0.0552 & -0.0672 & 0.6099
\end{array}\right], \mathbf{O}_{m}=\left[\begin{array}{ccc}
0.2731 & -0.0886 & 0.3411 \\
0.0542 & 0.5649 & 0.1034 \\
-3.0502 & -0.1992 & 2.6870
\end{array}\right]
$$
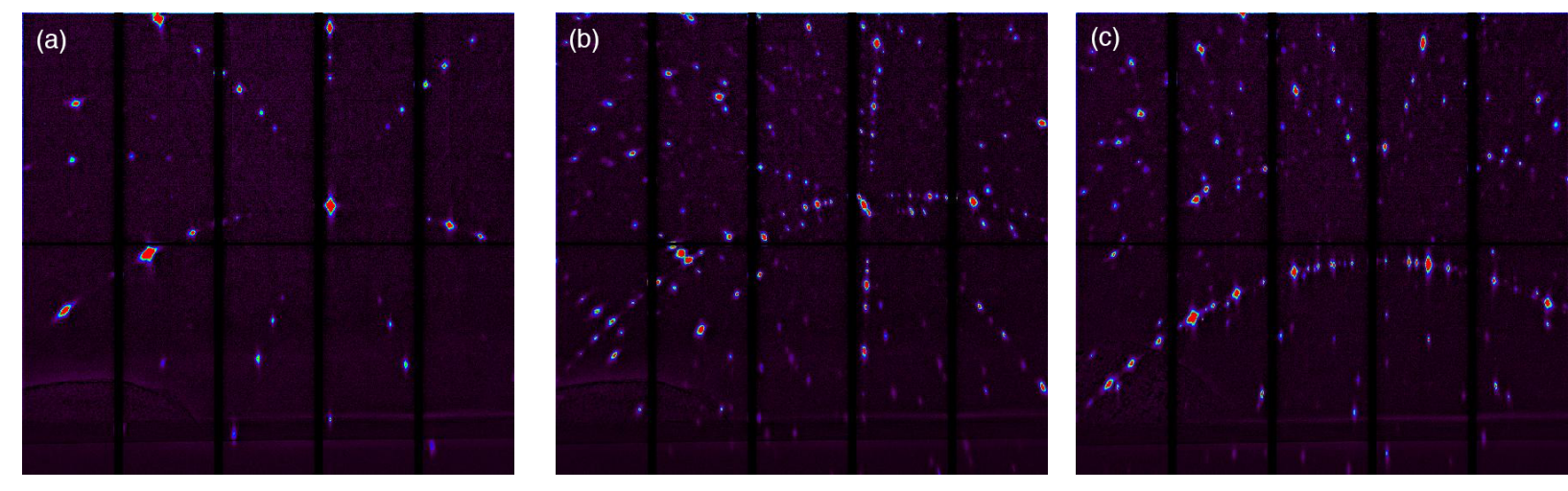

Figure 6: Typical Laue patterns of $\mathrm{Zn}_{45} \mathrm{Au}_{30} \mathrm{Cu}_{25}$ at the same position of the sample: (a) austenite; (b) mixed phases; (c) martensite 


\subsection{Calculation of orientation relationships}

For a 3-dimensional structural transformation from $\mathcal{L}\left(\mathbf{E}_{A}\right)$ to $\mathcal{L}\left(\mathbf{E}_{B}\right)$, if the algorithm gives the lattice correspondence matrix $\mathbf{L}$ associated with the least value of the distance function (4), we assume that the lattice vectors $\left(\mathbf{a}_{1}, \mathbf{a}_{2}, \mathbf{a}_{3}\right)=\mathbf{E}_{A} \mathbf{L}$ are parallel to the lattice vectors $\left(\mathbf{b}_{1}, \mathbf{b}_{2}, \mathbf{b}_{3}\right)=\mathbf{E}_{B}$ closely. Here we use the conventional notation "I" to represent this closeness, i.e., $\mathbf{E}_{B} \| \mathbf{E}_{A} \mathbf{L}$ and $\mathbf{E}_{B} \mathbf{L}^{-1} \| \mathbf{E}_{A}$.

For any $\mathbf{r} \in \mathcal{L}(\mathbf{E})$, we have $\mathbf{r}=\mathbf{E n}$ where $\mathbf{n} \in \mathbb{Z}^{3}$ is the crystallographic directional index, and $\mathbf{r}^{*}=\mathbf{E}^{-T} \mathbf{n}^{*}$ where $\mathbf{n}^{*}$ is the crystallographic planar index. By the closeness assumption, we have

$$
\begin{gathered}
\mathbf{n}_{B} \| \mathbf{L}^{-1} \mathbf{n}_{A}, \\
\mathbf{n}_{B}^{*} \| \mathbf{L}^{T} \mathbf{n}_{A}^{*}
\end{gathered}
$$

In the case of $\mathrm{Zn}_{45} \mathrm{Au}_{30} \mathrm{Cu}_{25}$ in Table 1, the algorithm indicates that there exist two lattice correspondences

$$
\mathbf{L}_{I}=\left[\begin{array}{ccc}
\frac{1}{2} & 0 & \overline{4} \\
0 & 1 & 0 \\
\frac{1}{2} & 0 & 5
\end{array}\right] \text {, and } \mathbf{L}_{I I}=\left[\begin{array}{ccc}
\frac{1}{2} & 0 & \frac{-9}{2} \\
0 & 1 & 0 \\
\frac{1}{2} & 0 & \frac{9}{2}
\end{array}\right]
$$

For cubic to monoclinic transformation there are 12 symmetry-related correspondence matrices. $\mathbf{L}_{I}$ and $\mathbf{L}_{I I}$ are chosen so as to match the crystallographic orientation from the X-ray Laue diffraction measurement [6].
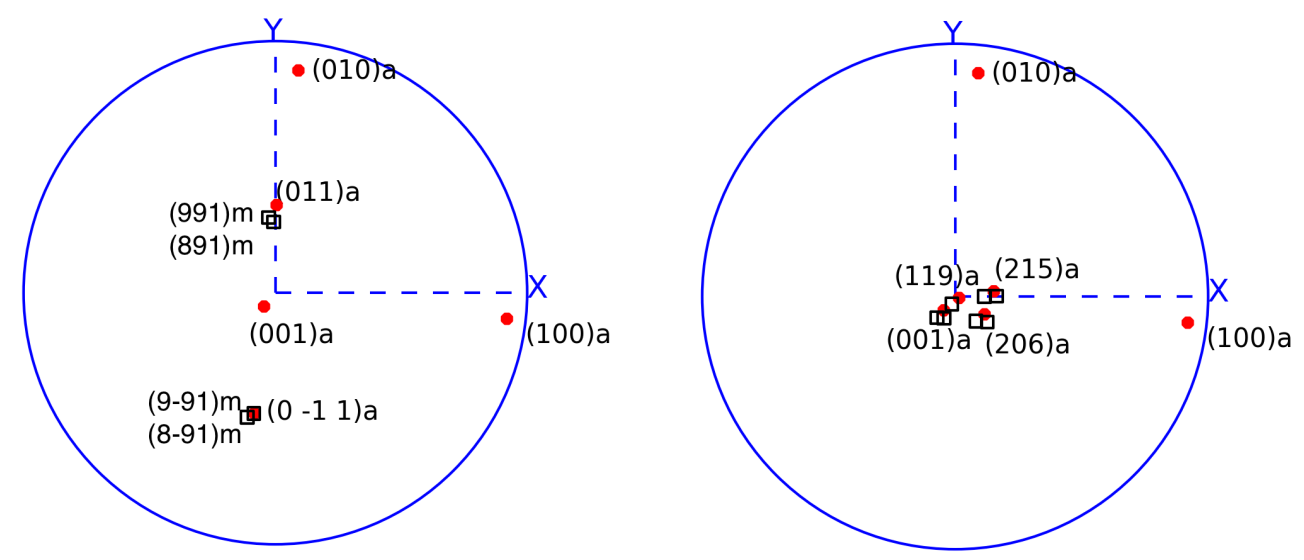

Figure 7: Stereographic projections of the $\mathrm{Zn}_{45} \mathrm{Au}_{30} \mathrm{Cu}_{25}$ from the orientation matrices measured by X-ray Laue diffraction: (a) crystallographic directions; (b) crystallographic planes. (red dots denote the cubic phase, black boxes denote the monoclinic phase.)

The correspondence matrices in (10) are not symmetry-related, both give small transformation strain, but can hardly be distinguished by X-ray Laue diffraction. For example, we choose crystallographic directions $\mathbf{n}_{A}=[0,1,1]_{a}, \quad[0,-1,1]_{a}$ and crystallographic planes $\mathbf{n}_{A}^{*}=(2,0,6)_{a},(0,0,1)_{a},(2,1,5)_{a},(1,1,9)_{a}$. By equation (8), the parallelisms given by $\mathbf{L}_{I}$ 
and $\mathbf{L}_{I I}$ are

$$
\begin{gathered}
\left.(2,0,6)_{\mathrm{a}}\left\|(2,0,11)_{\mathrm{m}},(0,0,1)_{\mathrm{a}}\right\|(1,0,10)_{\mathrm{m}},(2,1,5)_{\mathrm{a}}\|\mid\|, 2,34\right)_{\mathrm{m}},(1,1,9)_{\mathrm{a}} \|(5,1,41)_{\mathrm{m}} \\
{[0,1,1]_{\mathrm{a}}\left\|(8,9,1)_{\mathrm{m}},[0,-1,1]_{\mathrm{a}}\right\|[8,-9,1]_{\mathrm{m}}}
\end{gathered}
$$

and

$$
\begin{gathered}
\left.(2,0,6)_{\mathrm{a}}\left\|(2,0,9)_{\mathrm{m}}, \quad(0,0,1)_{\mathrm{a}}\right\|(1,0,9)_{\mathrm{m}},(2,1,5)_{\mathrm{a}} \| 7,2,27\right)_{\mathrm{m}},(1,1,9)_{\mathrm{a}} \|(5,1,36)_{\mathrm{m}} \\
{[0,1,1]_{\mathrm{a}}\left\|(9,9,1)_{\mathrm{m}},[0,-1,1]_{\mathrm{a}}\right\|[9,-9,1]_{\mathrm{m}}}
\end{gathered}
$$

respectively. Fig. 7 shows the stereographic projections by X-ray Laue diffraction for both cubic (red dots) and monoclinic (black boxes) phases. As seen from Fig. 7, these two transformation mechanisms cannot be distinguished.

We conclude from this comparison that the micro-Laue method as described above, in conjunction with the algorithm presented here, can be a useful tool for inferring orientation relationships and therefore transformation stretch tensors, but, in the case of $\mathrm{Zn}_{45} \mathrm{Au}_{30} \mathrm{Cu}_{25}$, it is not able to unequivocally declare one mechanism identified by the algorithm as being preferable to the other. The most interesting situation, and, in our opinion, the most likely, is that both mechanisms occur in $\mathrm{Zn}_{45} \mathrm{Au}_{30} \mathrm{Cu}_{25}$. Both transformation stretch tensors closely satisfy the cofactor conditions. The simultaneous occurrence of both mechanisms would imply that there would be two sets of 12 variants available to the material, each with a great many conditions of compatibility closely satisfied both between individual variants and also with austenite.

\section{Acknowledgments}

We thank Liping Liu, Robert Kohn, Kaushik Bhattacharya, Anton Mühlemann and Konstantinos Koumatos for helpful discussions during the preparation of this work. XC, YS, and RDJ acknowledge the support of the MURI project Managing the Mosaic of Microstructure (FA9550-12-1-0458, administered by AFOSR), NSF-PIRE (OISE-0967140), ONR (N0001414-1-0714) and AFOSR FA9550-15-1-0207. The Advanced Light Source is supported by the Director, Office of Science, Office of Basic Energy Sciences, of the U.S. Department of Energy under Contract No. DE-AC02-05CH11231. XS and NT would like to thank Alastair McDowell and Scott DiMaggio for their assistance on the technical design and construction of the sample heating stage.

[1] M. Bailey and C. J. Brown. The crystal structure of Terephthalic Acid. Acta Cryst., 22:387, 1967.

[2] E. C. Bain. The nature of martensite. Trans. AIME, 70(1):25, 1924.

[3] J. M. Ball and R. D. James. Fine phase mixtures as minimizers of energy. Arch. Ration. Mech. Anal., 100(1):13, 1987.

[4] J. M. Ball and R. D. James. Proposed experimental tests of a theory of fine microstructure and the two-well problem. Phil. Trans.: Phys. Sci. Eng., 338(1650):389, 1992. 
[5] J. M. Ball and R. D. James. Incompatible sets of gradients and metastability. Arch. Ration. Mech. Anal., pages 1-54, 2015.

[6] K. Bhattacharya. Microstructure of martensite: why it forms and how it gives rise to the shape-memory effect. Oxford Series on Materials Modeling. Oxford University Press, 2003.

[7] J. S. Bowles and C. M. Wayman. The bain strain, lattice correspondences, and deformations related to martensitic transformation. Metall. Trans., 3:1113, 1972.

[8] X. Chen, S. Cao, T. Ikeda, V. Srivastava, G. J. Snyder, D. Schryvers, and R. D. James. A weak compatibility condition for precipitation with application to the microstructure of $\mathrm{PbTe}-\mathrm{Sb}_{2} \mathrm{Te}_{3}$ thermoelectrics. Acta Mater., 59(15):6124, 2011.

[9] X. Chen, V. Srivastava, V. Dabade, and R. D. James. Study of the cofactor conditions: conditions of supercompatibility between phases. J. Mech. Phys. Solids, 61(12):2566, 2013 .

[10] C. Chluba, W. Ge, R. L. de Miranda, J. Strobel, L. Kienle, E. Quandt, and M. Wuttig. Ultralow-fatigue shape memory alloy films. Science, 348(6238):1004-1007, 2015.

[11] C. H. Chu. Hysteresis and microstructures: A study of biaxial loading on compound twins of copper-aluminium-nickel single crystals. PhD thesis, University of Minnesota, 193.

[12] J. Cui, Y. S. Chu, O. O. Famodu, Y. Furuya, J. Hattrick-Simpers, R. D. James, A. Ludwig, S. Thienhaus, M. Wuttig, Z. Zhang, and I. Takeuchi. Combinatorial search of thermoelastic shape-memory alloys with extremely small hysteresis width. Nature Mater., $5(4): 286-290,2006$.

[13] J. Cui, Y. Wu, J. Muehlbauer, Y. Hwang, R. Radermacher, S. Fackler, M. Wuttig, and I. Takeuchi. Demonstration of high efficiency elastocaloric cooling with large $\Delta \mathrm{T}$ using NiTi wires. Appl. Phys. Lett., 101(7):073904, 2012.

[14] R. J. Davey, S. J. Maginn, S. J. Andrews, A. M. Buckley, D. Cottler, P. Dempsey, J. E. Rout, D. R. Stanley, and A. Taylor. Stabilization of a metastable crystalline phase by twinning. Nature, 366:248, 1993.

[15] M. J. Duggin and W. A. Rachinger. A martensitic transformation in a gold-copper-zinc alloy. Acta Metall., 12:1015, 1964.

[16] R. Fonda and H. Jones. Microstructure, crystallography, and shape memory effect in equiatomic NbRu. Mater. Sci. Eng. A, 273-275:275, 1999.

[17] X. Huang, G. J. Ackland, and K. M. Rabe. Crystal structures and shape-memory behaviour of NiTi. Nature Materials, 2:307, 2003.

[18] T. Ikeda, V. A. Ravi, and G. J. Snyder. Formation of $\mathrm{Sb}_{2} \mathrm{Te}_{3}$ Widmanstätten precipitates in thermoelectric PbTe. Acta Mater., 57:666, 2009. 
[19] R. D. James. Taming the temperamental metal transformation. Science, 348(6238):968969, 2015.

[20] B. Kang and G. Ceder. Battery materials for ultrafast charging and discharging. Nature, 458(190), 2009.

[21] Kaufmann, S. and Rößler, U. K. and Heczko, O. and Wuttig, M. and Buschbeck, J. and Schultz, L. and Fähler, S. Adaptive modulations of martensites. Phys. Rev. Lett., 104:145702, 2010.

[22] K. M. Knowles and D. A. Smith. The crystallography of the martensitic transformation in equiatomic nickel-titanium. Acta Metall., 29:101, 1980.

[23] K. M. Knowles and D. A. Smith. The nature of the parent-martensite interface in Titanium-Manganese. Acta Metall., 29:1445, 1981.

[24] M. Kunz, N. Tamura, K. Chen, A. A. MacDowell, R. Celestre, M. Church, S. Fakra, E. Domning, J. Glossinger, J. Kirschman, G. Morrison, D. Plate, B. Smith, T. Warwick, V. Yashchuk, H. Padmore, and E. Ustundag. A dedicated superbend x-ray microdiffraction beamline for materials-, geo- and environmental sciences at the Advanced Light Source. Review of Scientific Instruments, 80(3):035108, 2009.

[25] G. V. Kurdjumov, V. A. Lobodyuk, and L. G. Khandros. The form of martensitic crystals and the orientation of the interphase boundaries in cualni alloy. Soviet Phys. Cryst., 6:165, 1961.

[26] M. H. Li, P. Keller, B. Li, X. Wang, and M. Brunet. Light-driven side-on nematic elastomer actuators. Adv. Mater., 15(7-8):569, 2003.

[27] J. Liu, T. Gottschall, K. P. Skokov, J. D., Moore, and O. Gutfleisch. Giant magnetocaloric effect driven by structural transitions. Nature Mater., 11(7):620-626, 2012.

[28] W. M. Lomer. The $\beta \rightarrow \alpha$ transformation in Uranium-1.4 atomic percent Chromium alloy. Inst. Metals Monogr., (18):243, 1955.

[29] M. W. Louie, M. Kislitsyn, K. Bhattacharya, and S. M. Haile. Phase transformation and hysteresis behavior in $\mathrm{Cs}_{1-x} \mathrm{Rb}_{x} \mathrm{H}_{2} \mathrm{PO}_{4}$. Solid State Ion., 181(3-4):173 - 179, 2010.

[30] A. Mühlemann and K. Koumatos. On the optimality of the Bain strain in steels and general lattice transformations. preprint, 2015.

[31] K. Otsuka, T. Ohba, M. Tokonami, and C. M. Wayman. New discription of long period stacking structures of martensites in beta-phase alloys. Scripta Metallurgica et Materialia, 29:1359-1364, 1993.

[32] K. Otsuka, T. Sawamura, and K. I. Shimizu. Crystal structure and internal defects of equiatomic TiNi martensite. Phys. Stat. Sol. (a), 5:457, 1971.

[33] K. Otsuka and K. I. Shimizu. Morphology and crystallography of thermoelastic Cu-Al-Ni martensite analyzed by the phenomenological theory. Trans. JIM, 15:103, 1974. 
[34] K. Otsuka and C. M. Wayman. Shape memory materials. Cambridge University Press, 1999.

[35] M. Pitteri and G. Zanzotto. Continuum models for phase transitions and twinning in crystals. Chapman and Hall/CRC, 2010.

[36] Y. Song, K. Bhatti, V. Srinivasan, C. Leighton, and R. D. James. Thermodynamics of energy conversion via first order phase transformation in low hysteresis magnetic materials. Energy Environ. Sci., 6(4):1315, 2013.

[37] Y. Song, X. Chen, V. Dabade, T. W. Shield, and R. D. James. Enhanced reversibility and unusual microstructure of a phase-transforming material. Nature, 502:85, 2013.

[38] V. Srivastava, X. Chen, and R. D. James. Hysteresis and unusual magnetic properties in the singular heusler alloy $\mathrm{Ni}_{45} \mathrm{Co}_{5} \mathrm{Mn}_{40} \mathrm{Sn}_{10}$. Appl. Phys. Lett., 97(1):014101, 2010.

[39] V. Srivastava, Y. Song, K. Bhatti, and R. D. James. The direct conversion of heat to electricity using multiferroic alloys. Adv. Energy Mater., 1(1):97-104, 2011.

[40] T. Tadaki and K. Shimizu. Trans. Japan Inst. Metals, 11:44, 1970.

[41] N. Tamura. XMAS: a versatile tool for analyzing synchrotron x-ray microdiffraction data. Imperial College Press (London), 2014.

[42] C. M. Wayman. Introduction to the Crystallography of Martensitic Transformation. Macmillan, 1964.

[43] R. Zarnetta, R. Takahashi, M. L. Young, A. Savan, Y. Furuya, S. Thienhaus, B. Maaß, M. Rahim, J. Frenzel, H. Brunken, Y. S. Chu, V. Srivastava, R. D. James, I. Takeuchi, G. Eggeler, and A. Ludwig. Identification of quaternary shape memory alloys with near-zero thermal hysteresis and unprecedented functional stability. Adv. Funct. Mater., 20(12):1917-1923, 2010. 